\title{
Explicit solutions of initial value problems for systems of linear Riemann-Liouville fractional differential equations with constant delay
}

\author{
S. Hristova ${ }^{1 *}$, Ravi Agarwal ${ }^{2,3}$ and D. O'Regan ${ }^{4}$
}

"Correspondence:

snehri@gmail.com

'University of Plovdiv "Paisii

Hilendarski", Plovdiv, Bulgaria

Full list of author information is

available at the end of the article

\begin{abstract}
A system of linear Riemann-Liouville fractional differential equations with constant delay is studied. The initial condition is set up similar to the case of the ordinary derivative. Explicit formulas for the solutions are obtained for various initial functions.

MSC: $34 \mathrm{~A} 08 ; 34 \mathrm{~A} 37$

Keywords: Riemann-Liouville fractional derivative; Constant delay; Initial value problem; Systems of linear fractional equations; Explicit solution; q-matrix exponential function
\end{abstract}

\section{Introduction}

Usually fractional differential equations are considered as a generalization of ordinary differential equations, and they have been applied as more appropriate models of real world problems in engineering, physics, finance, etc. [9, 22]. The applications of fractional calculus have been growing, including anomalous diffusion [5], viscoelastic mechanics [6], control system [12], petroleum engineering [19], multi-strain tuberculosis model [23], and many other branches of physics and engineering. A good collection of different fractional models applied to thermodiffusion, thermodynamics, mechanics, and viscoelasticity is given in the book [24].

In many processes, such as chemical processes (behaviors in chemical kinetics), technical processes (electric, pneumatic, and hydraulic networks), biosciences (heredity in population dynamics), economics (dynamics of business cycles), a delay is observed. With the combination of both fractional derivative and time delay, the topic of fractional order delay differential equations is enjoying growing interest among mathematicians and physicists (see, for example, [11] for delayed feedback control).

One of the main qualitative problems is connected with obtaining explicit formulas for the solutions, especially in the case of linear equations. The generalized Mittag-Leffler function with matrix arguments is applied for systems of linear Caputo fractional differential equations (see [8]). Recently, there have been developments on seeking the explicit formula of solutions to delay Caputo fractional differential equations. Li and Wang [13]

(c) The Author(s) 2020. This article is licensed under a Creative Commons Attribution 4.0 International License, which permits use sharing, adaptation, distribution and reproduction in any medium or format, as long as you give appropriate credit to the original author(s) and the source, provide a link to the Creative Commons licence, and indicate if changes were made. The images or other third party material in this article are included in the article's Creative Commons licence, unless indicated otherwise in a credit line to the material. If material is not included in the article's Creative Commons licence and your intended use is not permitted by statutory regulation or exceeds the permitted use, you will need to obtain permission directly from the copyright holder. To view a copy of this licence, visit http://creativecommons.org/licenses/by/4.0/. 
studied the linear homogenous Caputo fractional delay differential equations and gave a representation of the solution. Also, in $[15,17]$ representations of the solution of linear non-homogeneous Caputo fractional delay differential equations are provided.

However, little is known regarding Riemann-Liouville (RL) fractional differential equations with delays. For some related contributions about RL factional differential equations, one can refer to previous works $[1,3]$. Note that linear systems of RL fractional differential equations without any delay are studied in [18] and explicit formulas for the solutions are obtained. RL fractional differential equations with delays are not well studied. We mention the papers $[14,16]$ where the lower bound of the RL fractional derivative coincides with the left side end of the initial interval, but we note that this does not correspond to the idea in the case of delay differential equations with ordinary derivatives and the idea of the initial value problem of RL fractional differential equations.

In this paper we study initial value problems of systems of linear RL fractional differential equations with constant delay of the type

$$
{ }_{0}^{\mathrm{RL}} D_{t}^{q} X(t)=A X(t)+B X(t-\tau)+F(t) \quad \text { for } t \in(0, T],
$$

where $A, B$ are constant matrices, $\tau>0$ is a constant delay, and $T \leq \infty$. Similar to the case of the ordinary derivative, the differential equation is given to the right of the initial time interval. It requires the lower bound of the RL fractional derivative to coincide with the right side end of the initial interval (usually this point is zero). Note that in this case any solution of an initial problem (IVP) with RL fractional derivatives is not continuous at the initial point. That is why RL fractional delay differential equations are convenient for modeling a process with impulsive types of initial conditions. These types of processes can be found in physics, chemistry, engineering, biology, and economics. To determine the law of the initial impulsive reaction, we need to add to the usual initial condition (for example, $x(t)=\phi(t)$ on the initial interval $[-\tau, 0], \tau>0$ is the delay) a fractional condition. This conclusion is based on the results obtained in [9] and [21] concerning the physical interpretation of RL fractional derivatives and initial conditions which include derivatives of the same kind. Based on the above, we set up appropriate IVPs for RL linear fractional differential equations with the lower limit of the $R L$ derivative equal to the right side point of the initial interval, i.e., we study initial conditions of the type

$$
X(t)=G(t) \quad \text { for } t \in[-\tau, 0]
$$

and

$$
\left.{ }_{0} I_{t}^{1-q} X(t)\right|_{t=0}=\lim _{t \rightarrow 0+} \frac{1}{\Gamma(1-q)} \int_{0}^{t} \frac{X(s)}{(t-s)^{q}} d s=C,
$$

where $C$ is a constant vector.

Explicit formulas for the solutions of initial value problems with both zero and nonzero initial functions are obtained. Also, the cases of homogeneous as well as non-homogeneous equations are studied. In the case $A=0$ the explicit formulas are comparatively easy to be applied, and in the case $A \neq 0$ the $q$-matrix functions (or the matrix MittagLeffler functions) are used. The scalar case of the linear RL fractional differential equations with the above mentioned initial conditions is studied in [2] and explicit solutions are 
obtained. Note that in [2] the Mittag-Leffler function is applied in all formulas for the explicit solutions. In the case of systems and vector functions, the Mittag-Leffler function is not applicable and it is replaced with the $q$-matrix exponential function defined and used in [4]. The application of this function not only leads to more complicated calculations but also to new formulas for the exacts solutions.

\section{Preliminary notes on fractional derivatives and equations}

Let $m \in L_{1}^{\text {loc }}\left(\left[t_{0}, T\right], \mathbb{R}\right)$ and $t_{0}, T \geq 0: t_{0}<T \leq \infty$ (in the case $T=\infty$ the intervals $\left(t_{0}, T\right]$ and $\left[t_{0}, T\right]$ are $\left(t_{0}, T\right)$ and $\left[t_{0}, T\right)$, respectively). In this paper we use the following definitions for fractional derivatives and integrals:

- Riemann-Liouville fractional integral of order $q \in(0,1)[7,20]$

$$
{ }_{t_{0}} I_{t}^{q} m(t)=\frac{1}{\Gamma(q)} \int_{t_{0}}^{t} \frac{m(s)}{(t-s)^{1-q}} d s, \quad t \in\left[t_{0}, T\right]
$$

where $\Gamma(\cdot)$ is the gamma function.

Note that sometimes the notation ${ }_{t_{0}} D_{t}^{-q} m(t)={ }_{t_{0}} I_{t}^{q} m(t)$ is used.

- Riemann-Liouville fractional derivative of order $q \in(0,1)[7,20]$

$$
{ }_{t_{0}}^{\mathrm{RL}} D_{t}^{q} m(t)=\frac{d}{d t}\left(t_{0} I_{t}^{1-q} m(t)\right)=\frac{1}{\Gamma(1-q)} \frac{d}{d t} \int_{t_{0}}^{t}(t-s)^{-q} m(s) d s, \quad t \in\left[t_{0}, T\right] .
$$

We give fractional integrals and RL fractional derivatives of some elementary functions which will be used later.

Proposition 1 For $t>t_{0}$ and $\beta>0$, the following equalities are true:

$$
\begin{aligned}
& { }_{t_{0}}^{\mathrm{RL}} D_{t}^{q}\left(t-t_{0}\right)^{\beta}=\frac{\Gamma(1+\beta)}{\Gamma(1+\beta-q)}\left(t-t_{0}\right)^{\beta-q}, \\
& t_{0} I_{t}^{1-q}\left(t-t_{0}\right)^{\beta-1}=\frac{\Gamma(\beta)}{\Gamma(1+\beta-q)}\left(t-t_{0}\right)^{\beta-q}, \\
& t_{0} I_{t}^{1-q}\left(t-t_{0}\right)^{q-1}=\Gamma(q), \\
& { }_{t_{0}}^{\mathrm{RL}} D_{t}^{q}\left(t-t_{0}\right)^{q-1}=0 .
\end{aligned}
$$

For $m \in L_{1}^{\text {loc }}\left(\left[t_{0}, T\right], \mathbb{R}^{n}\right), m=\left(m_{1}, m_{2}, \ldots, m_{n}\right)^{T}$, we use

$$
{ }_{t_{0}}^{\mathrm{RL}} D_{t}^{q} m(t)=\left({ }_{t_{0}}^{\mathrm{RL}} D_{t}^{q} m_{1}(t),{ }_{t_{0}}^{\mathrm{RL}} D_{t}^{q} m_{2}(t), \ldots, t_{0}{ }_{t_{0}} D_{t}^{q} m_{n}(t)\right)^{T}
$$

and

$$
{ }_{t_{0}} I_{t}^{q} m(t)=\left({ }_{t_{0}} I_{t}^{q} m_{1}(t),{ }_{t_{0}} I_{t}^{q} m_{2}(t), \ldots, t_{0} I_{t}^{q} m_{n}(t)\right)^{T} .
$$

Let $M_{n \times n}(\mathbb{R})$ be the set of all matrices $A=\left\{a_{i j}\right\}_{i, j=1}^{n}$ with $a_{i j} \in \mathbb{R}$. We will use the notation $I$ for the unit matrix from $M_{n \times n}(\mathbb{R})$. For any matrix $A \in M_{n \times n}(\mathbb{R})$, we will use the notation $A^{0}=I$ and define the $q$-matrix exponential function [4] by

$$
e_{q}^{A\left(t-t_{0}\right)}=\left(t-t_{0}\right)^{q-1} \sum_{k=0}^{\infty} A^{k} \frac{\left(t-t_{0}\right)^{k q}}{\Gamma((k+1) q)}, \quad t \geq t_{0},
$$


and the Mittag-Leffler matrix function by

$$
E_{q}\left(A\left(t-t_{0}\right)^{q}\right)=\sum_{k=0}^{\infty} A^{k} \frac{\left(t-t_{0}\right)^{k q}}{\Gamma((k+1) q)}
$$

Therefore,

$$
e_{q}^{A\left(t-t_{0}\right)}=\left(t-t_{0}\right)^{q-1} E_{q}\left(A\left(t-t_{0}\right)^{q}\right) .
$$

The definitions of the initial condition for systems of fractional differential equations with RL-derivatives are based on the following result for the linear RL matrix fractional equation:

$$
{ }_{t_{0}}^{\mathrm{RL}} D_{t}^{q} X(t)=A X(t), \quad t \in\left(t_{0}, T\right]
$$

with $X \in L_{1}^{\text {loc }}\left(\left[t_{0}, T\right], \mathbb{R}^{n}\right)$ and $A \in M_{n \times n}(\mathbb{R})$.

Proposition 2 (Theorem $3.1[25]$ ) Let $q \in(0,1)$. Then problem (1) with the initial condition

$$
\left.t_{0} I_{t}^{1-q} X(t)\right|_{t=t_{0}}:=\lim _{t \rightarrow t_{0}+} t_{0} I_{t}^{1-q} m(t)=C, \quad C \in M_{n \times n}(\mathbb{R})
$$

is equivalent to problem (1) with the initial condition

$$
\lim _{t \rightarrow t_{0}+}\left[\left(t-t_{0}\right)^{q-1} X(t)\right]=\frac{C}{\Gamma(q)} .
$$

Remark 1 According to Proposition 2, it is enough to study one of the initial conditions (2) or (3). Following this result, we will study only the initial condition of type (2).

The explicit formula of the initial value problem (IVP) (1), (2) is given by (see Theorem $3.2[25])$

$$
X(t)=e_{q}^{A\left(t-t_{0}\right)} C
$$

In the case of a system of non-homogeneous fractional differential equations with RLderivatives

$$
{ }_{t_{0}}^{\mathrm{RL}} D_{t}^{q} X(t)=A X+F(t)(t), \quad t \in\left(t_{0}, T\right]
$$

with $F \in C\left(\left[t_{0}, T\right], \mathbb{R}^{n}\right)$, the explicit formula of IVP (5), (2) is given by (see Theorem 3 and Remark 1 [18])

$$
X(t)=e_{q}^{A\left(t-t_{0}\right)} C+\int_{t_{0}}^{t} e_{q}^{A(t-s)} F(s) d s \quad \text { for } t \in\left(t_{0}, T\right] .
$$

In the case of a scalar linear RL fractional differential equation, we have the following result. 
Proposition 3 (Example 4.1 [10]) The solution of the Cauchy type problem

$$
\begin{aligned}
& { }_{t_{0}}^{\mathrm{RL}} D_{t}^{q} x(t)=\lambda x(t)+f(t) \quad \text { for } t \in\left(t_{0}, T\right], \\
& \left.{ }_{t_{0}} I_{t}^{1-q} x(t)\right|_{t=t_{0}}=b
\end{aligned}
$$

with $b \in \mathbb{R}, f \in\left(\left[t_{0}, T\right], \mathbb{R}\right)$ has the following form (formula 4.1.14 [10]):

$$
x(t)=\frac{b}{\left(t-t_{0}\right)^{1-q}} E_{q, q}\left(\lambda\left(t-t_{0}\right)^{q}\right)+\int_{t_{0}}^{t}(t-s)^{q-1} E_{q, q}\left(\lambda(t-s)^{q}\right) f(s) d s
$$

where $E_{p, q}(z)=\sum_{j=0}^{\infty} \frac{z^{j}}{\Gamma(j p+q)}$ is the Mittag-Leffler function with two parameters (see, for example, [20]).

In the case of a system of RL fractional differential equations with constant coefficients, we have the following result, which is a special case of Theorem 3 [18].

Proposition 4 ([18]) The solution to the initial value problem for the system

$$
\begin{aligned}
& { }_{t_{0}}^{\mathrm{RL}} D_{t}^{q} X(t)=A X(t)+F(t) \quad \text { for } t \in\left(t_{0}, T\right], \\
& \left.{ }_{t_{0}} I_{t}^{1-q} X(t)\right|_{t=t_{0}}=C
\end{aligned}
$$

with $A \in M_{n \times n}(\mathbb{R}), F \in\left(\left[t_{0}, T\right], \mathbb{R}^{n}\right)$ has the following form:

$$
X(t)=e_{q}^{A\left(t-t_{0}\right)} C+\int_{t_{0}}^{t} e_{q}^{A(t-s)} F(s) d s, \quad t \in\left(t_{0}, T\right]
$$

or its equivalent form

$$
X(t)=\left(t-t_{0}\right)^{q-1} E_{q}\left(A\left(t-t_{0}\right)^{q}\right) C+\int_{t_{0}}^{t}(t-s)^{q-1} E_{q}\left(A(t-s)^{q}\right) F(s) d s, \quad t \in\left(t_{0}, T\right] .
$$

\section{Explicit formula for the solutions of scalar linear RL fractional equations with delays and zero initial values}

Throughout the paper we assume $\sum_{i=n}^{l}(*)=0$ for the integers $n, l, n>l$.

\subsection{Homogeneous linear RL fractional differential equation}

Consider the system of linear Riemann-Liouville fractional differential equations with constant delay (HFrDE):

$$
{ }_{0}^{\mathrm{RL}} D_{t}^{q} X(t)=B X(t-\tau) \quad \text { for } t \in(0, T]
$$

where $q \in(0,1), B \in M_{n \times n}(\mathbb{R}), B=\left\{b_{i j}\right\}, \tau>0$ is a real constant, $X=\left(X_{1}, X_{2}, \ldots, X_{n}\right)^{T}, X_{k} \in$ $\mathbb{R}, k=1,2, \ldots, n$.

Remark 2 Without loss of generality we assume that there exists a natural number $N \leq \infty$ such that $T=(N+1) \tau$. 
We will consider the zero initial value

$$
X(t)=0 \quad \text { for } t \in[-\tau, 0] \text {, }
$$

and

$$
\left.{ }_{0} I_{t}^{1-q} X(t)\right|_{t=0}=\lim _{t \rightarrow 0+} \frac{1}{\Gamma(1-q)} \int_{0}^{t} \frac{X(s)}{(t-s)^{q}} d s=\mathbf{1},
$$

with $\mathbf{1}=(1,1, \ldots, 1)^{T}$.

Remark 3 Note that the IVP for $\operatorname{HFrDE}(10)$, (11) with the zero fractional initial condition, i.e., $\lim _{t \rightarrow 0+}\left(t^{1-q} X(t)\right)=0$, has only a zero solution.

Theorem 1 The solution of IVP (10), (11), (12) is given by

$$
X(t)=\sum_{i=0}^{n} \frac{B^{i}}{\Gamma((i+1) q)}(t-i \tau)^{(i+1) q-1} \mathbf{1}, \quad t \in(n \tau,(n+1) \tau], n=0,1,2, \ldots, N
$$

Proof Let $t \in(0, \tau]$. Then from (10) we have RL fractional differential equations ${ }_{0}^{\mathrm{RL}} D_{t}^{q} x \times$ $X_{i}(t)=0$ for $t \in(0, \tau], i=1,2, \ldots, n$, whose solution is given by

$$
X(t)=\frac{t^{q-1}}{\Gamma(q)} \mathbf{1}, \quad t \in(0, \tau]
$$

since from Proposition 1 we have ${ }_{0} I^{1-q} t^{q-1}=\Gamma(q)$, i.e., $\left.{ }_{0} I_{t}^{1-q} X(t)\right|_{t=0}=\mathbf{1}$ and

$$
{ }_{0}^{\mathrm{RL}} D_{t}^{q} \frac{t^{q-1}}{\Gamma(q)}=0
$$

Let $t \in(\tau, 2 \tau]$. Then from (10), (14) we have the following system of RL fractional equations:

$$
{ }_{0}^{\mathrm{RL}} D_{t}^{q} X(t)=B \frac{(t-\tau)^{q-1}}{\Gamma(q)} \mathbf{1} \text { for } t \in(\tau, 2 \tau]
$$

Then the solution of IVP (10), (11), (12) is given by

$$
X(t)=\frac{t^{q-1}}{\Gamma(q)} \mathbf{1}+\frac{B}{\Gamma(2 q)}(t-\tau)^{2 q-1} \mathbf{1}, \quad t \in(\tau, 2 \tau]
$$

Indeed, from Proposition 1 with $\beta=2 q-1$, we have for $t \in(\tau, 2 \tau]$ that

$$
\begin{aligned}
{ }_{0}^{\mathrm{RL}} D_{t}^{q} X(t)= & \frac{1}{\Gamma(1-q)} \frac{d}{d t}\left(\int_{0}^{\tau}(t-s)^{-q} X(s) d s+\int_{\tau}^{t}(t-s)^{-q} X(s) d s\right) \\
= & \frac{1}{\Gamma(1-q)} \frac{d}{d t} \int_{0}^{\tau}(t-s)^{-q} \frac{s^{q-1}}{\Gamma(q)} d s \mathbf{1} \\
& +\frac{1}{\Gamma(1-q)} \frac{d}{d t} \int_{\tau}^{t}(t-s)^{-q}\left(\frac{s^{q-1}}{\Gamma(q)} \mathbf{1}+\frac{B}{\Gamma(2 q)}(s-\tau)^{2 q-1} \mathbf{1}\right) d s
\end{aligned}
$$




$$
\begin{aligned}
= & \frac{1}{\Gamma(1-q)} \frac{d}{d t} \int_{0}^{t}(t-s)^{-q} \frac{s^{q-1}}{\Gamma(q)} d s \mathbf{1} \\
& +\frac{B}{\Gamma(2 q) \Gamma(1-q)} \frac{d}{d t} \int_{\tau}^{t}(t-s)^{-q}(s-\tau)^{2 q-1} d s \mathbf{1} \\
= & \frac{B}{\Gamma(q)}(t-\tau)^{q-1} \mathbf{1} .
\end{aligned}
$$

Therefore, $X(t)$ satisfies (16) for $t \in(\tau, 2 \tau]$.

Let $t \in(2 \tau, 3 \tau]$. Then from (10), (14), (17) we have

$$
{ }_{0}^{\mathrm{RL}} D_{t}^{q} X(t)=B \frac{(t-\tau)^{q-1}}{\Gamma(q)} \mathbf{1}+\frac{B^{2}}{\Gamma(2 q)}(t-2 \tau)^{2 q-1} \mathbf{1} \quad \text { for } t \in(2 \tau, 3 \tau] .
$$

Then the solution of IVP (10), (11), (12) is given by

$$
X(t)=\frac{t^{q-1}}{\Gamma(q)} \mathbf{1}+\frac{B}{\Gamma(2 q)}(t-\tau)^{2 q-1} \mathbf{1}+\frac{B^{2}}{\Gamma(3 q)}(t-2 \tau)^{3 q-1} \mathbf{1}, \quad t \in(2 \tau, 3 \tau]
$$

since from Proposition 1 with $\beta=2 q-1$ and the equality

$$
\frac{d}{d t} \int_{a}^{t}(t-s)^{-q}(s-a)^{k q-1} d s=\frac{(t-a)^{(k-1) q-1} \Gamma(1-q) \Gamma(k q)}{\Gamma((k-1) q)}
$$

we have for $t \in(2 \tau, 3 \tau]$ that

$$
\begin{aligned}
& { }_{0}^{\mathrm{RL}} D_{t}^{q} X(t)=\frac{1}{\Gamma(1-q)} \frac{d}{d t}\left(\int_{0}^{\tau}(t-s)^{-q} X(s) d s+\int_{\tau}^{2 \tau}(t-s)^{-q} X(s) d s\right. \\
& \left.+\int_{2 \tau}^{t}(t-s)^{-q} X(s) d s\right) \\
& =\frac{1}{\Gamma(1-q)} \frac{d}{d t} \int_{0}^{\tau}(t-s)^{-q} \frac{s^{q-1}}{\Gamma(q)} d s \mathbf{1} \\
& +\frac{1}{\Gamma(1-q)} \frac{d}{d t} \int_{\tau}^{2 \tau}(t-s)^{-q}\left(\frac{s^{q-1}}{\Gamma(q)} \mathbf{1}+\frac{B}{\Gamma(2 q)}(s-\tau)^{2 q-1} \mathbf{1}\right) d s \\
& +\frac{1}{\Gamma(1-q)} \frac{d}{d t} \int_{2 \tau}^{t}(t-s)^{-q} \\
& \times\left(\frac{s^{q-1}}{\Gamma(q)} \mathbf{1}+\frac{B}{\Gamma(2 q)}(s-\tau)^{2 q-1} \mathbf{1}+\frac{B^{2}}{\Gamma(3 q)}(s-2 \tau)^{3 q-1} \mathbf{1}\right) d s \\
& =\frac{1}{\Gamma(1-q)} \frac{d}{d t} \int_{0}^{t}(t-s)^{-q} \frac{s^{q-1}}{\Gamma(q)} d s \mathbf{1} \\
& +\frac{B}{\Gamma(2 q) \Gamma(1-q)} \frac{d}{d t} \int_{\tau}^{t}(t-s)^{-q}(s-\tau)^{2 q-1} \mathbf{1} d s \\
& +\frac{B^{2}}{\Gamma(3 q) \Gamma(1-q)} \frac{d}{d t} \int_{2 \tau}^{t}(t-s)^{-q}(s-2 \tau)^{3 q-1} \mathbf{1} d s \\
& =\frac{B}{\Gamma(q)}(t-\tau)^{q-1} \mathbf{1}+\frac{B^{2}}{\Gamma(2 q)}(t-2 \tau)^{2 q-1} \mathbf{1} \text {. }
\end{aligned}
$$

Therefore, $X(t)$, defined by (19), satisfies (19) for $t \in(2 \tau, 3 \tau]$. 
Continue this process and the claim is established.

Remark 4 In the scalar case $n=1$ the system of RL delay fractional equations (10) is reduced to a scalar linear delay RL fractional equation, the vector $\mathbf{1}$ is reduced to the constant 1 , and formula (13) coincides with equation (7) [2].

\subsection{Non-homogeneous linear RL fractional differential equation}

Consider non-homogeneous scalar linear Riemann-Liouville fractional differential equations with constant delay (NFrDE):

$$
{ }_{0}^{\mathrm{RL}} D_{t}^{q} X(t)=B X(t-\tau)+F(t) \quad \text { for } t \in(0, T],
$$

with the zero initial condition (11) and fractional condition

$$
\left.{ }_{0} I_{t}^{1-q} X(t)\right|_{t=0}=0
$$

where $F \in C\left(\mathbb{R}_{+}, \mathbb{R}^{n}\right), F=\left(F_{1}, F_{2}, \ldots, F_{n}\right)^{T}, \tau>0$ is a real constant, and $B \in M_{n \times n}$.

Using a direct proof, we will obtain an explicit formula for the solution of IVP (23), (11), (24).

Theorem 2 The solution of IVP (23), (11), (24) is given by

$$
\begin{aligned}
X(t) & =\sum_{i=0}^{n} \frac{B^{i}}{\Gamma((i+1) q)} \int_{i \tau}^{t}(t-s)^{(i+1) q-1} F(s-i \tau) d s, \\
t & \in(n \tau,(n+1) \tau], n=0,1, \ldots, N .
\end{aligned}
$$

Proof Let $t \in(0, \tau]$. Use the variation of constants method and we will search for solutions in the form

$$
X(t)=\int_{0}^{t} \frac{(t-s)^{q-1}}{\Gamma(q)} K(s) d s
$$

where $K \in C\left([0, \tau], \mathbb{R}^{n}\right), K=\left(K_{1}, K_{2}, \ldots, K_{n}\right)^{T}$ is the unknown function to be obtained. According to the initial condition (11), we have $X(t-\tau)=0$ for $t \in[0, \tau]$ and

$$
{ }_{0}^{\mathrm{RL}} D_{t}^{q} X_{i}(t)=F_{i}(t) \quad \text { for } t \in(0, \tau], i=1,2, \ldots, n \text {. }
$$

Then, applying $\int_{\xi}^{t}(t-s)^{-q}(s-\xi)^{q-1} d s=\Gamma(1-q) \Gamma(q)$, we obtain for $t \in(0, \tau]$ that

$$
\begin{aligned}
{ }_{0}^{\mathrm{RL}} D_{t}^{q} X_{i}(t) & =\frac{1}{\Gamma(1-q)} \frac{d}{d t} \int_{0}^{t}(t-s)^{-q} \int_{0}^{s} \frac{(s-\xi)^{q-1}}{\Gamma(q)} K_{i}(\xi) d \xi d s \\
& =\frac{1}{\Gamma(1-q)} \frac{d}{d t} \int_{0}^{t} \int_{\xi}^{t}(t-s)^{-q} \frac{(s-\xi)^{q-1}}{\Gamma(q)} K(\xi) d s d \xi \\
& =\frac{d}{d t} \int_{0}^{t} K_{i}(\xi)\left(\frac{1}{\Gamma(1-q)} \int_{\xi}^{t}(t-s)^{-q} \frac{(s-\xi)^{q-1}}{\Gamma(q)} d s\right) d \xi \\
& =\frac{d}{d t} \int_{0}^{t} K_{i}(\xi) d \xi=K_{i}(t) .
\end{aligned}
$$


From (27) and (28) we get $K_{i}(t) \equiv F_{i}(t), i=1,2, \ldots, n$, i.e., the solution $X(t)$ of IVP (23), (11), (24) for $t \in(0, \tau]$ is given by

$$
X(t)=\int_{0}^{t} \frac{(t-s)^{q-1}}{\Gamma(q)} F(s) d s, \quad t \in(0, \tau]
$$

Note that it is easy to check the validity of condition (24) for the solution $X(t)$ defined by (29).

Let $t \in(\tau, 2 \tau]$. Use the variation of constants method and we will search for solutions in the form

$$
X(t)=\int_{0}^{t} \frac{(t-s)^{q-1}}{\Gamma(q)} F(s) d s+\frac{B}{\Gamma(2 q)} \int_{\tau}^{t}(t-s)^{2 q-1} K(s) d s \quad \text { for } t \in(\tau, 2 \tau]
$$

where $K \in C\left([\tau, 2 \tau], \mathbb{R}^{n}\right), K=\left(K_{1}, K_{2}, \ldots, K_{n}\right)^{T}$ is the unknown function to be obtained.

Then, according to (23), (2), and $X(t-\tau)=\int_{0}^{t-\tau} \frac{(t-\tau-s)^{q-1}}{\Gamma(q)} F(s) d s$, for $t \in(2 \tau, 3 \tau]$, we have

$$
\begin{aligned}
{ }_{0}^{\mathrm{RL}} D_{t}^{q} X(t) & =B \int_{0}^{t-\tau} \frac{(t-\tau-s)^{q-1}}{\Gamma(q)} F(s) d s+F(t) \\
& =B \int_{\tau}^{t} \frac{(t-s)^{q-1}}{\Gamma(q)} F(s-\tau) d s+F(t) \quad \text { for } t \in(\tau, 2 \tau] .
\end{aligned}
$$

Also, applying $-q \Gamma(-q)=\Gamma(1-q)$, equality ${ }_{0} I_{t-\xi}^{1-(q+1)}(t-\xi)^{2 q-1}=\frac{\Gamma(2 q)}{\Gamma(q)}(t-\xi)^{q-1}$ (see Proposition 1), (21) with $a=\xi, k=2$, and

$$
\begin{aligned}
\frac{d}{d t} & \int_{\tau}^{t} K(\xi)\left(\int_{\xi}^{t}(t-s)^{-q}(s-\xi)^{2 q-1} d s\right) d \xi \\
& =\int_{\tau}^{t} K(\xi) \frac{d}{d t}\left(\int_{\xi}^{t}(t-s)^{-q}(s-\xi)^{2 q-1} d s\right) d \xi \\
& =\frac{\Gamma(1-q) \Gamma(2 q)}{\Gamma(q)} \int_{\tau}^{t} K(\xi)(t-\xi)^{q-1} d \xi,
\end{aligned}
$$

from (2) and (30) we obtain for $t \in(\tau, 2 \tau]$ that

$$
\begin{aligned}
{ }_{0}^{\mathrm{RL}} D_{t}^{q} X(t)= & \frac{1}{\Gamma(1-q)} \frac{d}{d t}\left(\int_{0}^{\tau}(t-s)^{-q} X(s) d s+\int_{\tau}^{t}(t-s)^{-q} X(s) d s\right) \\
= & \frac{1}{\Gamma(1-q)} \frac{d}{d t}\left(\int_{0}^{\tau}(t-s)^{-q} \int_{0}^{s} \frac{(s-\xi)^{q-1}}{\Gamma(q)} F(\xi) d \xi d s\right. \\
& +\int_{\tau}^{t}(t-s)^{-q} \int_{0}^{s} \frac{(s-\xi)^{q-1}}{\Gamma(q)} F(\xi) d \xi d s \\
& \left.+\int_{\tau}^{t}(t-s)^{-q} \frac{B}{\Gamma(2 q)} \int_{\tau}^{s}(s-\xi)^{2 q-1} K(\xi) d \xi d s\right) \\
= & \frac{1}{\Gamma(1-q)} \frac{d}{d t}\left(\int_{0}^{t}(t-s)^{-q} \int_{0}^{s} \frac{(s-\xi)^{q-1}}{\Gamma(q)} F(\xi) d \xi d s\right. \\
& \left.+\frac{B}{\Gamma(2 q)} \int_{\tau}^{t} K(\xi) \int_{\xi}^{t}(t-s)^{-q}(s-\xi)^{2 q-1} d s d \xi\right)
\end{aligned}
$$




$$
\begin{aligned}
& =F(t)+\frac{B}{\Gamma(2 q) \Gamma(1-q)} \frac{d}{d t} \int_{\tau}^{t} K(\xi)\left(\int_{\xi}^{t}(t-s)^{-q}(s-\xi)^{2 q-1} d s\right) d \xi \\
& =F(t)+B \int_{\tau}^{t} K(\xi) \frac{(t-\xi)^{q-1}}{\Gamma(q)} d \xi .
\end{aligned}
$$

From (31) and (33) we get $K(s)=F(s-\tau), s \in[\tau, t]$, and

$$
X(t)=\int_{0}^{t} \frac{(t-s)^{q-1}}{\Gamma(q)} F(s) d s+\frac{B}{\Gamma(2 q)} \int_{\tau}^{t}(t-s)^{2 q-1} F(s-\tau) d s \quad \text { for } t \in(\tau, 2 \tau] .
$$

Let $t \in(2 \tau, 3 \tau]$. Use the variation of constants method and we will search for solutions in the form

$$
\begin{aligned}
X(t)= & \int_{0}^{t} \frac{(t-s)^{q-1}}{\Gamma(q)} f(s) d s+\frac{B}{\Gamma(2 q)} \int_{\tau}^{t}(t-s)^{2 q-1} F(s-\tau) d s \\
& +\frac{B^{2}}{\Gamma(3 q)} \int_{2 \tau}^{t}(t-s)^{3 q-1} K(s) d s \quad \text { for } t \in(2 \tau, 3 \tau],
\end{aligned}
$$

where $K \in C\left([2 \tau, 3 \tau], \mathbb{R}^{n}\right), K=\left(K_{1}, K_{2}, \ldots, K_{n}\right)^{T}$ is the unknown function to be obtained.

Then, according to (23), (2), and (34), we have for $t \in(2 \tau, 3 \tau]$ that

$$
\begin{aligned}
{ }_{0}^{\mathrm{RL}} D_{t}^{q} x(t)= & B \int_{0}^{t-\tau} \frac{(t-\tau-s)^{q-1}}{\Gamma(q)} F(s) d s \\
& +\frac{B^{2}}{\Gamma(2 q)} \int_{\tau}^{t-\tau}(t-\tau-s)^{2 q-1} F(s-\tau) d s+F(t) \\
= & B \int_{\tau}^{t} \frac{(t-s)^{q-1}}{\Gamma(q)} F(s-\tau) d s+\frac{B^{2}}{\Gamma(2 q)} \int_{2 \tau}^{t}(t-s)^{2 q-1} F(s-2 \tau) d s+F(t) .
\end{aligned}
$$

Similar to (33) we obtain

$$
\begin{aligned}
{ }_{0}^{\mathrm{RL}} D_{t}^{q} X(t)= & \frac{1}{\Gamma(1-q)} \frac{d}{d t}\left(\int_{0}^{t}(t-s)^{-q} \int_{0}^{s} \frac{(s-\xi)^{q-1}}{\Gamma(q)} F(\xi) d \xi d s\right. \\
& +\frac{B}{\Gamma(2 q)} \int_{\tau}^{t}(t-s)^{-q} \int_{\tau}^{s}(s-\xi)^{2 q-1} F(\xi-\tau) d \xi d s \\
& \left.+\frac{B^{2}}{\Gamma(3 q)} \int_{2 \tau}^{t}(t-s)^{-q} \int_{2 \tau}^{s}(s-\xi)^{3 q-1} K(\xi) d \xi d s\right) \\
= & F(t)+B \int_{\tau}^{t} \frac{(t-\xi)^{q-1}}{\Gamma(q)} F(\xi-\tau) d \xi \\
& +\frac{B^{2}}{\Gamma(3 q) \Gamma(1-q)} \int_{2 \tau}^{t} K(\xi) \frac{d}{d t}\left(\int_{\xi}^{t}(t-s)^{-q}(s-\xi)^{3 q-1} d s\right) d \xi \\
= & F(t)+B \int_{\tau}^{t} \frac{(t-\xi)^{q-1}}{\Gamma(q)} F(\xi-\tau) d \xi+B \int_{2 \tau}^{t} K(\xi) \frac{(t-\xi)^{2 q-1}}{\Gamma(2 q)} d \xi,
\end{aligned}
$$

i.e., from (36) and (37) we get $K(s)=F(s-2 \tau), s \in[2 \tau, t]$, and

$$
\begin{aligned}
X(t)= & \int_{0}^{t} \frac{(t-s)^{q-1}}{\Gamma(q)} F(s) d s+\frac{B}{\Gamma(2 q)} \int_{\tau}^{t}(t-s)^{2 q-1} F(s-\tau) d s \\
& +\frac{B^{2}}{\Gamma(3 q)} \int_{2 \tau}^{t}(t-s)^{3 q-1} F(s-2 \tau) d s \quad \text { for } t \in(2 \tau, 3 \tau] .
\end{aligned}
$$


Continue this process and the claim is established.

Remark 5 Note that the formula for the solution in the homogeneous case does not follow from the one in the non-homogeneous case because of fractional conditions (12), respectively, (24).

Remark 6 The explicit formula (25) of solution in Theorem 2 is a generalization of formula (20) [2] for the scalar case $n=1$.

\section{Explicit formula for the solutions of scalar linear RL fractional equations with delays and non-zero initial values}

Consider the linear non-homogeneous RL fractional differential equation (23) with nonzero initial value:

$$
\begin{aligned}
& X(t)=G(t) \quad \text { for } t \in[-\tau, 0], \\
& \left.{ }_{0} I_{t}^{1-q} X(t)\right|_{t=0}:=\lim _{t \rightarrow 0+}{ }_{0} I_{t}^{1-q} X(t)=\lim _{t \rightarrow 0+} \frac{1}{\Gamma(1-q)} \int_{0}^{t} \frac{X(s)}{(t-s)^{q}} d s=G(0),
\end{aligned}
$$

where $G \in C\left([-\tau, 0], \mathbb{R}^{n}\right), \max _{i=1,2, \ldots, n}\left|G_{i}(0)\right|<\infty$.

Remark 7 Note that the function $G(t)=t^{q-1} \mathbf{1}$ is not applicable in this case as an initial function.

Theorem 3 The solution of IVP (23), (39) is given by

$$
X(t)=\left\{\begin{aligned}
& G(t), \quad t \in[-\tau, 0] \\
& \sum_{k=0}^{n} \frac{B^{k} G(0)}{\Gamma((k+1) q)}(t-k \tau)^{(k+1) q-1} \\
& \quad+\sum_{k=0}^{n} \frac{B^{k}}{\Gamma((k+1) q)} \int_{k \tau}^{t}(t-s)^{(k+1) q-1} F(s-k \tau) d s \\
& \quad+\sum_{k=0}^{n-1} \frac{B^{k+1}}{\Gamma((k+1) q)} \int_{k \tau}^{(k+1) \tau}(t-s)^{(k+1) q-1} G(s-(k+1) \tau) d s \\
& \quad+\frac{B^{n}}{\Gamma((n+1) q)} \int_{n \tau}^{t}(t-s)^{(n+1) q-1} G(s-(n+1) \tau) d s, \\
& t \in(n \tau,(n+1) \tau], n=3,4, \ldots, N .
\end{aligned}\right.
$$

Proof Define the function $P \in C\left([0, T], \mathbb{R}^{n}\right), P=\left(P_{1}, P_{2}, \ldots, P_{n}\right)$, by

$$
P(t)=\left\{\begin{array}{l}
B G(t-\tau)+F(t), \quad t \in[0, \tau], \\
\frac{(t-\tau)^{q-1}}{\Gamma(q)} B G(0)+\frac{B}{\Gamma(q)} \int_{\tau}^{t}(t-s)^{q-1}(B G(s-2 \tau)+F(s-\tau)) d s+F(t), \quad t \in(\tau, 2 \tau], \\
\frac{(t-\tau)^{q-1}}{\Gamma(q)} B G(0)+\frac{(t-2 \tau)^{2 q-1}}{\Gamma(2 q)} B^{2} G(0)+\frac{B}{\Gamma(q)} \int_{\tau}^{t}(t-s)^{q-1} F(s-\tau) d s \\
\quad+\frac{B^{2}}{\Gamma(q)} \int_{\tau}^{2 \tau}(t-s)^{q-1} G(s-2 \tau) d s \\
\quad+\frac{B^{2}}{\Gamma(2 q)} \int_{2 \tau}^{t}(B G(s-3 \tau)+F(s-2 \tau))(t-s)^{2 q-1} d s+F(t), \quad t \in(2 \tau, 3 \tau], \\
\ldots, \\
\quad \sum_{k=1}^{n+1} \frac{B^{k} G(0)}{\Gamma(k q)}(t-k \tau)^{k q-1}+\sum_{k=1}^{n} \frac{B^{k}}{\Gamma(k q)} \int_{k \tau}^{t}(t-s)^{k q-1} F(s-k \tau) d s \\
\quad+\sum_{k=1}^{n-1} \frac{B^{k+1}}{\Gamma(k q)} \int_{k \tau}^{(k+1) \tau}(t-s)^{k q-1} G(s-k \tau) d s \\
\quad+\frac{B^{n+1}}{\Gamma(n q)} \int_{n \tau}^{t-\tau}(t-s)^{n q-1} G(s-n \tau) d s+F(t), \\
\quad t \in(n \tau,(n+1) \tau], n=0,1,2, \ldots, N .
\end{array}\right.
$$


Let $t \in(0, \tau]$. Then, from system (23) and initial condition (39), we have

$$
\begin{aligned}
& { }_{0}^{\mathrm{RL}} D_{t}^{q} X(t)=B G(t-\tau)+F(t) \text { for } t \in(0, \tau] \\
& \left.{ }_{0} I_{t}^{1-q} X(t)\right|_{t=0}=G(0) .
\end{aligned}
$$

Therefore, we have

$$
\begin{aligned}
& { }_{0}^{\mathrm{RL}} D_{t}^{q} X_{i}(t)=P_{i}(t) \quad \text { for } t \in(0, \tau], \\
& \left.{ }_{0} I_{t}^{1-q} X_{i}(t)\right|_{t=0}=G_{i}(0), \quad i=1,2, \ldots, n .
\end{aligned}
$$

According to Proposition 3 with $\lambda=0$ and the equality $E_{q, q}(0)=\frac{1}{\Gamma(q)}$, the solution of $(41)$ on $(0, \tau]$ is

$$
\begin{aligned}
X_{i}(t) & =\frac{G_{i}(0)}{\Gamma(q)} t^{q-1}+\frac{1}{\Gamma(q)} \int_{0}^{t}(t-s)^{q-1} P_{i}(s) d s \\
& =\frac{G_{i}(0)}{\Gamma(q)} t^{q-1}+\frac{\sum_{j=1}^{n} b_{i j}}{\Gamma(q)} \int_{0}^{t}(t-s)^{q-1} G_{j}(s-\tau) d s+\frac{1}{\Gamma(q)} \int_{0}^{t}(t-s)^{q-1} F_{i}(s) d s .
\end{aligned}
$$

Therefore, the solution of IVP (23), (39) on $(0, \tau]$ is given by

$$
X(t)=\frac{G(0)}{\Gamma(q)} t^{q-1}+\frac{1}{\Gamma(q)} \int_{0}^{t}(t-s)^{q-1}(B G(s-\tau)+F(s)) d s, \quad t \in(0, \tau]
$$

Let $t \in(\tau, 2 \tau]$. Then from (23), (39), and (42) we have the system

$$
{ }_{0}^{\mathrm{RL}} D_{t}^{q} X_{i}(t)=P_{i}(t), \quad t \in(\tau, 2 \tau], i=1,2, \ldots, n .
$$

According to Proposition 3 with $\lambda=0$, the equality $E_{q, q}(0)=\frac{1}{\Gamma(q)}$, and $\int_{\xi}^{t}(t-s)^{q-1}(s-$ $\xi)^{q-1} d s=\frac{\Gamma^{2}(q)}{\Gamma(2 q)}(t-\xi)^{2 q-1}$, the solution of $(44)$ on $(\tau, 2 \tau]$ is

$$
\begin{aligned}
X_{i}(t)= & \frac{G_{i}(0)}{\Gamma(q)} t^{q-1}+\frac{1}{\Gamma(q)} \int_{0}^{\tau}(t-s)^{q-1} P_{i}(s) d s+\frac{1}{\Gamma(q)} \int_{\tau}^{t}(t-s)^{q-1} P_{i}(s) d s \\
= & \frac{G_{i}(0)}{\Gamma(q)} t^{q-1}+\frac{1}{\Gamma(q)} \int_{0}^{t}(t-s)^{q-1} F_{i}(s) d s+\frac{1}{\Gamma(q)} \int_{0}^{\tau}(t-s)^{q-1} \sum_{j=1}^{n} b_{i j} G_{j}(s-\tau) d s \\
& +\frac{1}{\Gamma(q)} \int_{\tau}^{t}(t-s)^{q-1} \frac{\sum_{j=1}^{n} b_{i j} G_{j}(0)}{\Gamma(q)}(s-\tau)^{q-1} d s \\
& +\frac{1}{\Gamma(q)} \int_{\tau}^{t}(t-s)^{q-1} \sum_{j=1}^{n} b_{i j} \frac{\sum_{k=1}^{n} b_{j k}}{\Gamma(q)} \int_{\tau}^{s}(s-\xi)^{q-1} G_{k}(\xi-2 \tau) d \xi d s \\
& +\frac{1}{\Gamma(q)} \int_{\tau}^{t}(t-s)^{q-1} \frac{\sum_{j=1}^{n} b_{i j}}{\Gamma(q)} \int_{\tau}^{s}(s-\xi)^{q-1} F_{j}(\xi-\tau) d \xi d s \\
= & \frac{G_{i}(0)}{\Gamma(q)} t^{q-1}+\frac{1}{\Gamma(q)} \int_{0}^{t}(t-s)^{q-1} F_{i}(s) d s+\frac{1}{\Gamma(q)} \int_{0}^{\tau}(t-s)^{q-1} \sum_{j=1}^{n} b_{i j} G_{j}(s-\tau) d s \\
& +\frac{\sum_{j=1}^{n} b_{i j} G_{j}(0)}{\Gamma^{2}(q)} \int_{\tau}^{t}(t-s)^{q-1}(s-\tau)^{q-1} d s
\end{aligned}
$$




$$
\begin{aligned}
& +\frac{1}{\Gamma^{2}(q)} \int_{\tau}^{t}\left(\sum_{j=1}^{n} b_{i j} \sum_{k=1}^{n} b_{j k} G_{k}(\xi-2 \tau)\right) \int_{\xi}^{t}(t-s)^{q-1}(s-\xi)^{q-1} d s d \xi \\
& +\frac{1}{\Gamma^{2}(q)} \int_{\tau}^{t}\left(\sum_{j=1}^{n} b_{i j} F_{j}(\xi-\tau)\right) \int_{\xi}^{t}(t-s)^{q-1}(s-\xi)^{q-1} d s d \xi \\
& =\frac{G_{i}(0)}{\Gamma(q)} t^{q-1}+\frac{1}{\Gamma(q)} \int_{0}^{t}(t-s)^{q-1} F_{i}(s) d s+\frac{1}{\Gamma(q)} \int_{0}^{\tau}(t-s)^{q-1} \sum_{j=1}^{n} b_{i j} G_{j}(s-\tau) d s \\
& +\frac{1}{\Gamma(2 q)} \sum_{j=1}^{n} b_{i j} G_{j}(0)(t-\tau)^{2 q-1} \\
& +\frac{1}{\Gamma(2 q)} \int_{\tau}^{t}\left(\sum_{j=1}^{n} b_{i j} \sum_{k=1}^{n} b_{j k} G_{k}(\xi-2 \tau)+\sum_{j=1}^{n} b_{i j} F_{j}(\xi-\tau)\right)(t-\xi)^{2 q-1} d \xi .
\end{aligned}
$$

Therefore, from equation (45), $i=1,2, \ldots, n$, the solution of system $(23)$ on $(\tau, 2 \tau]$ is

$$
\begin{aligned}
X(t)= & \frac{G(0)}{\Gamma(q)} t^{q-1}+\frac{B G(0)}{\Gamma(2 q)}(t-\tau)^{2 q-1} \\
& +\frac{1}{\Gamma(q)} \int_{0}^{t}(t-s)^{q-1} F(s) d s+\frac{B}{\Gamma(2 q)} \int_{\tau}^{t}(t-s)^{2 q-1} F(s-\tau) d s \\
& +\frac{B}{\Gamma(q)} \int_{0}^{\tau}(t-s)^{q-1} G(s-\tau) d s+\frac{B^{2}}{\Gamma(2 q)} \int_{\tau}^{t}(t-s)^{2 q-1} G(s-2 \tau) d s, \\
& t \in(\tau, 2 \tau] .
\end{aligned}
$$

Continue this process and the claim is established.

Special case: In the homogeneous case when $F(t) \equiv 0$, the solution of IVP (10), (39) is given by

$$
X(t)=\left\{\begin{array}{l}
G(t), \quad t \in[-\tau, 0] \\
\sum_{k=0}^{n} \frac{B^{k} G(0)}{\Gamma((k+1) q)}(t-k \tau)^{(k+1) q-1} \\
\quad+\sum_{k=0}^{n-1} \frac{B^{k+1}}{\Gamma((k+1) q)} \int_{k \tau}^{(k+1) \tau}(t-s)^{(k+1) q-1} G(s-(k+1) \tau) d s \\
\quad+\frac{B^{n}}{\Gamma((n+1) q)} \int_{n \tau}^{t}(t-s)^{(n+1) q-1} G(s-(n+1) \tau) d s \\
\quad t \in(n \tau,(n+1) \tau], n=0,1,2, \ldots, N .
\end{array}\right.
$$

\section{Explicit formula for the solutions of the general scalar linear RL fractional equations with delays and non-zero initial values}

\subsection{Zero initial function}

Consider the system of non-homogeneous linear Riemann-Liouville fractional differential equations with constant delay:

$$
{ }_{0}^{\mathrm{RL}} D_{t}^{q} X(t)=A X(t)+B X(t-\tau)+F(t) \quad \text { for } t \in(0, T],
$$

with the initial conditions

$$
X(t)=0, \quad t \in[-\tau, 0],
$$




$$
\left.{ }_{0} I_{t}^{1-q} X(t)\right|_{t=0}=C
$$

where $X \in \mathbb{R}^{n}, F \in C\left(\mathbb{R}_{+}, \mathbb{R}^{n}\right), A, B \in M_{n \times n}(\mathbb{R}), \tau>0$ is a real constant, $C \in \mathbb{R}^{n}$.

Theorem 4 The solution of IVP (47), (48), (49) is given by

$$
X(t)=\left\{\begin{array}{l}
0 \quad \text { for } t \in(-\tau, 0], \\
e_{q}^{A t} C+\int_{0}^{t} e_{q}^{A(t-s)} F(s) d s, \quad \text { for } t \in(0, \tau], \\
e_{q}^{A t} C+\int_{0}^{t} e_{q}^{A(t-s)} F(s) d s \\
\quad+\sum_{k=1}^{n} \int_{k \tau}^{t} e_{q}^{A\left(t-s_{1}\right)} B \int_{k \tau}^{s_{1}} e_{q}^{A\left(s_{1}-s_{2}\right)} B \int_{k \tau}^{s_{2}} e_{q}^{A\left(s_{2}-s_{3}\right)} B \\
\quad \times \cdots \times \int_{k \tau}^{s_{k}} e_{q}^{A\left(s_{k-1}-s_{k}\right)} B\left(e_{q}^{A\left(s_{k}-k \tau\right)} C\right. \\
\left.\quad+\int_{k \tau}^{s_{k-1}} e_{q}^{A\left(s_{k}-\xi\right)} F(\xi-k \tau) d \xi\right) d s_{k} d s_{k-1} \cdots d s_{3} d s_{2} d s_{1} \\
\quad \text { for } t \in(n \tau,(n+1) \tau], n=1,2, \ldots, N .
\end{array}\right.
$$

Proof Define the function $P \in C\left([0, T], \mathbb{R}^{n}\right), P=\left(P_{1}, P_{2}, \ldots, P_{n}\right)$, by

$$
P(t)=\left\{\begin{array}{l}
F(t), \quad t \in[0, \tau], \\
B e_{q}^{A(t-\tau)} C+B \int_{\tau}^{t} e_{q}^{A(t-s)} F(s-\tau) d s+F(t), \quad t \in(\tau, 2 \tau], \\
B e_{q}^{A(t-\tau)} C+\int_{\tau}^{t} B e_{q}^{A(t-s)} F(s-\tau) d s \\
\quad+\int_{2 \tau}^{t} B e_{q}^{A(t-s)} B e_{q}^{A(s-2 \tau)} C d s \\
\quad+\int_{2 \tau}^{t} \int_{2 \tau}^{s} B e_{q}^{A(t-s)} B e_{q}^{A(s-\xi)} F(\xi-2 \tau) d \xi d s+F(t), \quad t \in(2 \tau, 3 \tau], \\
\quad, \quad \\
B e_{q}^{A(t-\tau)} C+\int_{0}^{t} e_{q}^{A(t-\tau-s)} F(s) d s \\
\quad+\sum_{k=1}^{n} \int_{k \tau}^{t-\tau} e_{q}^{A\left(t-\tau-s_{1}\right)} B \int_{k \tau}^{s_{1}} e_{q}^{A\left(s_{1}-s_{2}\right)} B \int_{k \tau}^{s_{2}} e_{q}^{A\left(s_{2}-s_{3}\right)} B \\
\quad \times \cdots \times \int_{k \tau}^{s_{k}} e_{q}^{A\left(s_{k-1}-s_{k}\right)} B\left(e_{q}^{A\left(s_{k}-k \tau\right)} C\right. \\
\left.\quad+\int_{k \tau}^{s_{k-1}} e_{q}^{A\left(s_{k}-\xi\right)} F(\xi-k \tau) d \xi\right) d s_{k} d s_{k-1} \cdots d s_{3} d s_{2} d s_{1}+F(t), \\
\quad t \in(n \tau,(n+1) \tau], n=3,4, \ldots, N .
\end{array}\right.
$$

Let $t \in(0, \tau]$. Then from equation (47) we have for $t \in(0, \tau]$ the equation

$$
{ }_{0}^{\mathrm{RL}} D_{t}^{q} X(t)=A X(t)+P(t)
$$

with initial condition (49).

According to Proposition 4, the solution of (50), (49) is

$$
X(t)=e_{q}^{A t} C+\int_{0}^{t} e_{q}^{A(t-s)} F(s) d s, \quad t \in(0, \tau]
$$

Let $t \in(\tau, 2 \tau]$. Then from (47), (48), (49), and (51) we have that system (50) is satisfied on $(\tau, 2 \tau]$, and therefore, the solution of (50), (49) according to Proposition 4 is

$$
\begin{aligned}
X(t) & =e_{q}^{A t} C+\int_{0}^{t} e_{q}^{A(t-s)} P(s) d s \\
& =e_{q}^{A t} C+\int_{0}^{\tau} e_{q}^{A(t-s)} P(s) d s+\int_{\tau}^{t} e_{q}^{A(t-s)} P(s) d s
\end{aligned}
$$




$$
\begin{aligned}
= & e_{q}^{A t} C+\int_{0}^{t} e_{q}^{A(t-s)} F(s) d s \\
& +\int_{\tau}^{t} e_{q}^{A(t-s)} B\left(e_{q}^{A(s-\tau)} C+\int_{\tau}^{s} e_{q}^{A(s-\xi)} F(\xi-\tau) d \xi\right) d s .
\end{aligned}
$$

Let $t \in(2 \tau, 3 \tau]$. Then from (47), (48), (49), and (52) we have that system (50) is satisfied on $(2 \tau, 3 \tau]$, and therefore, the solution of (50), (49) according to Proposition 4 is

$$
\begin{aligned}
X(t)= & e_{q}^{A t} C+\int_{0}^{\tau} e_{q}^{A(t-s)} P(s) d s+\int_{\tau}^{2 \tau} e_{q}^{A(t-s)} P(s) d s+\int_{2 \tau}^{t} e_{q}^{A(t-s)} P(s) d s \\
= & e_{q}^{A t} C+\int_{0}^{t} e_{q}^{A(t-s)} F(s) d s \\
& +\int_{\tau}^{2 \tau} e_{q}^{A(t-s)} B e_{q}^{A(s-\tau)} C d s+\int_{\tau}^{2 \tau} \int_{\tau}^{s} e_{q}^{A(t-s)} B e_{q}^{A(s-\xi)} F(\xi-\tau) d \xi d s \\
& +\int_{2 \tau}^{t} e_{q}^{A(t-s)} B e_{q}^{A(s-\tau)} C d s+\int_{2 \tau}^{t} \int_{\tau}^{s} e_{q}^{A(t-s)} B e_{q}^{A(s-\sigma)} F(\sigma-\tau) d \sigma d s \\
& +\int_{2 \tau}^{t} \int_{2 \tau}^{s} e_{q}^{A(t-s)} B e_{q}^{A(s-\sigma)} B e_{q}^{A(\sigma-2 \tau)} C d \sigma d s \\
& +\int_{2 \tau}^{t} \int_{2 \tau}^{s} \int_{2 \tau}^{\sigma} e_{q}^{A(t-s)} B e_{q}^{A(s-\sigma)} B e_{q}^{A(\sigma-\xi)} F(\xi-2 \tau) d \xi d \sigma d s \\
= & e_{q}^{A t} C+\int_{0}^{t} e_{q}^{A(t-s)} F(s) d s \\
& +\int_{\tau}^{t} e_{q}^{A(t-s)} B\left(e_{q}^{A(s-\tau)} C+\int_{\tau}^{s} e_{q}^{A(s-\xi)} F(\xi-\tau) d \xi\right) d s \\
& +\int_{2 \tau}^{t} \int_{2 \tau}^{s} e_{q}^{A(t-s)} B e_{q}^{A(s-\sigma)} B\left(e_{q}^{A(\sigma-2 \tau)} C+\int_{2 \tau}^{\sigma} e_{q}^{A(\sigma-\xi)} F(\xi-2 \tau) d \xi\right) d \sigma d s .
\end{aligned}
$$

Continue this process.

Remark 8 In the case $\tau=0$ (no delay), $B=0$, and $F \equiv 0$, the system of RL fractional differential equations (47) is reduced to the one studied in [25] with initial condition (49), and the formula obtained in Theorem 4 reduces to the formula obtained in Theorem 3.2 [25].

Remark 9 In the case $\tau=0$ (no delay), $B=0$, the system of RL fractional differential equations (47) is reduced to the studied system (34) in [4] with initial condition (49), and the formula obtained in Theorem 4 reduces to the formula obtained in Theorem 3 [4].

Special case: In the homogeneous case when $F(t) \equiv 0$, the solution of IVP (47), (48), (49) is given by

$$
X(t)=\left\{\begin{array}{l}
0 \text { for } t \in(-\tau, 0], \\
e_{q}^{A t} C, \quad \text { for } t \in(0, \tau], \\
e_{q}^{A t} C+\sum_{k=1}^{n} \int_{k \tau}^{t} e_{q}^{A\left(t-s_{1}\right)} B \int_{k \tau}^{s_{1}} e_{q}^{A\left(s_{1}-s_{2}\right)} B \int_{k \tau}^{s_{2}} e_{q}^{A\left(s_{2}-s_{3}\right)} B \\
\quad \times \cdots \times \int_{k \tau}^{s_{k-1}} e_{q}^{A\left(s_{k-1}-s_{k}\right)} B e_{q}^{A\left(s_{k}-k \tau\right)} d s_{k} d s_{k-1} \cdots d s_{3} d s_{2} d s_{1} C \\
\quad \text { for } t \in(n \tau,(n+1) \tau], n=1,2, \ldots, N .
\end{array}\right.
$$




\subsection{Non-zero initial function}

Consider non-homogeneous scalar linear Riemann-Liouville fractional differential equations with constant delay (47) with the initial conditions

$$
\begin{aligned}
& X(t)=G(t), \quad t \in[-\tau, 0], \\
& \left.{ }_{0} I_{t}^{1-q} X(t)\right|_{t=0}=G(0),
\end{aligned}
$$

where $G \in C\left([-\tau, 0], \mathbb{R}^{n}\right)$.

Theorem 5 The solution of IVP (47), (54), (55) is given by

$$
X(t)=\left\{\begin{array}{l}
G(t) \quad \text { for } t \in(-\tau, 0], \\
e_{q}^{A t} G(0)+\int_{0}^{t} e_{q}^{A(t-s)} F(s) d s, \quad \text { for } t \in(0, \tau], \\
e_{q}^{A t} G(0)+\int_{0}^{t} e_{q}^{A(t-s)} F(s) d s \\
\quad+\sum_{k=1}^{n} \int_{k \tau}^{t} e_{q}^{A\left(t-s_{1}\right)} B \int_{k \tau}^{s_{1}} e_{q}^{A\left(s_{1}-s_{2}\right)} B \int_{k \tau}^{s_{2}} e_{q}^{A\left(s_{2}-s_{3}\right)} B \\
\quad \times \cdots \times \int_{k \tau}^{s_{k-1}} e_{q}^{A\left(s_{k-1}-s_{k}\right)} B\left(e_{q}^{A\left(s_{k}-k \tau\right)} G\left(s_{k}-k \tau\right)\right. \\
\left.\quad+\int_{k \tau}^{s_{k}} e_{q}^{A\left(s_{k}-\xi\right)} F(\xi-k \tau) d \xi\right) d s_{k} d s_{k-1} \cdots d s_{3} d s_{2} d s_{1} \\
\quad \text { fort } t \in(n \tau,(n+1) \tau], n=1,2, \ldots, N .
\end{array}\right.
$$

Proof The proof is similar to the one of Theorem 4, so we omit it.

Special case: In the homogeneous case when $F(t) \equiv 0$, the solution of IVP (47), (54), (55) is given by

$$
X(t)=\left\{\begin{array}{l}
G(t) \quad \text { for } t \in(-\tau, 0], \\
e_{q}^{A t} G(0), \quad \text { for } t \in(0, \tau], \\
e_{q}^{A t} G(0)+\sum_{k=1}^{n} \int_{k \tau}^{t} e_{q}^{A\left(t-s_{1}\right)} B \int_{k \tau}^{s_{1}} e_{q}^{A\left(s_{1}-s_{2}\right)} B \int_{k \tau}^{s_{2}} e_{q}^{A\left(s_{2}-s_{3}\right)} B \\
\quad \times \cdots \times \int_{k \tau}^{s_{k-1}} e_{q}^{A\left(s_{k-1}-s_{k}\right)} B e_{q}^{A\left(s_{k}-k \tau\right)} G\left(s_{k}-k \tau\right) d s_{k} d s_{k-1} \cdots d s_{3} d s_{2} d s_{1} \\
\text { for } t \in(n \tau,(n+1) \tau], n=1,2, \ldots, N .
\end{array}\right.
$$

Remark 10 Note that in the case the initial time 0 is replaced with arbitrary $t_{0}$, all the results in the paper are true with slight changes.

\section{Conclusions}

The formulas for the exact solutions are important tools in fractional models. Often it is quite complicated to find the exact solution for RL fractional differential equations even in the linear scalar case. In this paper we study various types of systems of linear RL fractional differential equations with constant delays. We set up an initial value problem in an appropriate way based on the physical meaning to initial conditions expressed in terms of Riemann-Liouville fractional derivatives or integrals [9]. Explicit formulas for the solutions of initial value problems with both zero and nonzero initial functions are obtained, and systems with homogeneous and non-homogeneous equations are studied. The $q$-matrix exponential function is successfully applied in explicit solutions [4]. 
The obtained formulas will be very helpful in the theoretical study of fractional models with RL fractional derivative, for linearization of multi-dimensional nonlinear models, for the monotone-iterative technique, and for systems of RL fractional differential equations with delays.

\section{Acknowledgements}

Not applicable.

\section{Funding}

The research is supported by the Bulgarian National Science Fund under Project KP-06-N32/7.

Availability of data and materials

Not applicable.

\section{Competing interests}

The authors declare that there is no conflict of interests regarding the publication of this paper.

\section{Authors' contributions}

All authors contributed equally to the writing of this paper. Furthermore, all authors carefully read and approved the final manuscript.

\section{Author details}

${ }^{1}$ University of Plovdiv "Paisii Hilendarski", Plovdiv, Bulgaria. ${ }^{2}$ Department of Mathematics, Texas A\&M

University-Kingsville, Kingsville, USA. ${ }^{3}$ Florida Institute of Technology, Melbourne, USA. ${ }^{4}$ School of Mathematics,

Statistics and Applied Mathematics, National University of Ireland, Galway, Ireland.

\section{Publisher's Note}

Springer Nature remains neutral with regard to jurisdictional claims in published maps and institutional affiliations.

Received: 26 January 2020 Accepted: 16 April 2020 Published online: 25 April 2020

\section{References}

1. Agarwal, R., Hristova, S., O'Regan, D.: Basic concepts of Riemann-Liouville fractional differential equations with non-instantaneous impulses. Symmetry 11, 614 (2019)

2. Agarwal, R., Hristova, S., O'Regan, D.: Explicit solutions of initial value problems for linear scalar Riemann-Liouville fractional differential equations with a constant delay. Mathematics 8, 32 (2020). https://doi.org/10.3390/math8010032

3. Agarwal, R., Hristova, S., O'Regan, D.: Exact solutions of linear Riemann-Liouville fractional differential equations with impulses. Rocky Mt. J. Math. (to appear). https://projecteuclid.org/euclid.rmjm/1576227809

4. Bonilla, B., Rivero, M., Trujillo, J.J.: On systems of linear fractional differential equations with constant coefficients. Appl. Math. Comput. 187, 68-78 (2007)

5. Chechkin, A.V., Gorenflo, R., Sokolov, I.M.: Fractional diffusion in inhomogeneous media. J. Phys. A, Math. Gen. 38(42), 679-684 (2005)

6. Coimbra, C.F.M.: Mechanics with variable-order differential operators. Ann. Phys. 12(11-12), 692-703 (2003)

7. Diethelm, K.: The Analysis of Fractional Differential Equations. Springer, Berlin (2010)

8. Duan, J.: System of linear fractional differential equations and the Mittag-Leffler functions with matrix variable. J. Phys. Conf. Ser. 1053, 012032 (2018). https://doi.org/10.1088/1742-6596/1053/1/012032

9. Heymans, N., Podlubny, I.: Physical interpretation of initial conditions for fractional differential equations with Riemann-Liouville fractional derivatives. Rheol. Acta 45, 765-771 (2006)

10. Kilbas, A.A., Srivastava, H.M., Trujillo, J.J.: Theory and Applications of Fractional Differential Equations. Elsevier, Amsterdam (2006)

11. Konishi, K., Kokame, H., Hara, N.: Delayed feedback control based on the act-and-wait concept. Nonlinear Dyn. 63, 513-519 (2011)

12. Kumar, P., Chaudhary, S.K.: Analysis of fractional order control system with performance and stability. Int. J. Eng. Sci. Technol. 9(5), 408-416 (2017)

13. Li, M., Wang, J.: Finite time stability of fractional delay differential equations. Appl. Math. Lett. 64, 170-176 (2017)

14. Li, M., Wang, J.R.: Representation of solution of a Riemann-Liouville fractional differential equation with pure delay. Appl. Math. Lett. 85, 118-124 (2018)

15. Li, M., Wang, J.R.: Exploring delayed Mittag-Leffler type matrix functions to study finite time stability of fractional delay differential equations. Appl. Math. Comput. 324, 254-265 (2018)

16. Li, M., Wang, J.R.: Finite time stability and relative controllability of Riemann-Liouville fractional delay differential equations. Math. Methods Appl. Sci. 42,6607-6623 (2019). https://doi.org/10.1002/mma.5765

17. Liang, C., Wang, J.R., O'Regan, D.: Representation of solution of a fractional linear system with pure delay. Appl. Math. Lett. 77, 72-78 (2018)

18. Matychyn, I.: Analytical solution of linear fractional systems with variable coefficients involving Riemann-Liouville and Caputo derivatives. Symmetry 11, 1366 (2019). https://doi.org/10.3390/sym 11111366

19. Obembe, A.D., Hossain, M.E., Abu-Khamsin, S.A.: Variable-order derivative time fractional diffusion model for heterogeneous porous media. J. Pet. Sci. Eng. 152, 391-405 (2017) 
20. Podlubny, I.: Fractional Differential Equations. Academic Press, San Diego (1999)

21. Podlubny, l.: Geometric and physical interpretation of fractional integration and fractional differentiation. Fract. Calc. Appl. Anal. 5(4), 367-387 (2002)

22. Saxena, R.K., Mathai, A.M., Haubold, H.J.: On fractional kinetic equations. Astrophys. Space Sci. 282, $281-287$ (2002)

23. Sweilam, N.H., Al-Mekhlafi, S.M.: Numerical study for multi-strain tuberculosis (TB) model of variable-order fractional derivatives. J. Adv. Res. 7(2), 271-283 (2016)

24. Tarasov, V:: Handbook of Fractional Calculus with Applications. Appl. in Physics, Part A, vol. 4. de Gruyter, Berlin (2019)

25. Vasundhara Devi, J., Nagamani, S., Jayalakshmi, K.: Solutions of Riemann-Liouville fractional matrix differential equations. J. Ramanujan Math. Soc. 5(1), 111-126 (2016)

\section{Submit your manuscript to a SpringerOpen ${ }^{\circ}$} journal and benefit from:

- Convenient online submission

- Rigorous peer review

- Open access: articles freely available online

- High visibility within the field

- Retaining the copyright to your article

Submit your next manuscript at $\gg$ springeropen.com 